Ricardo B Noal'

Ana M B Menezes'

Simone F Canani"

Fernando V Siqueira'

\section{Ronco habitual e apnéia obstrutiva observada em adultos: estudo de base populacional, Pelotas, RS}

\section{Habitual snoring and obstructive sleep apnea in adults: population- based study in Southern Brazil}

Programa de Pós-graduação em Epidemiologia. Faculdade de Medicina. Universidade Federal de Pelotas. Pelotas, RS, Brasil

" Hospital de Clínicas de Porto Alegre. Pelotas, RS, Brasil

Correspondência | Correspondence: Ricardo Bica Noal

R. Rua Marechal Deodoro 1160

96020-220 Pelotas, RS, Brasil

E-mail: ricardonoal@gmail.com

\section{RESUMO}

OBJETIVO: Estimar a prevalência de ronco habitual e apnéia obstrutiva observada e fatores associados.

MÉTODOS: Estudo transversal com amostra representativa de 3.136 adultos, com 20 anos ou mais, residentes em Pelotas, Rio Grande do Sul, em 2005. Informações sobre os desfechos e variáveis demográficas, socioeconômicas, comportamentais e antropométricas foram coletadas por meio de questionário. A análise estatística bruta e ajustada foi realizada utilizando-se teste exato de Fisher ou qui-quadrado de tendência linear e regressão de Poisson, respectivamente.

RESULTADOS: A prevalência encontrada de ronco habitual foi de 50,5\% (IC 95\%: 48,1;52,8) e de apnéia obstrutiva de 9,9\% (IC 95\%: 8,7;11,2). $\mathrm{Na}$ análise ajustada, o relato de ronco foi maior nos homens (Razão de Prevalências - $\mathrm{RP}=1,25$; IC 95\%: 1,16;1,34), nos idosos ( $\mathrm{RP}=1,62$; IC 95\%: 1,46;1,80), nos tabagistas ( $\mathrm{RP}=1,15$; IC 95\%: 1,07;1,25), nos alcoolistas ( $\mathrm{RP}=1,17$; IC 95\%: 1,03;1,31) e nos obesos (RP 1,71, IC95\% 1,55;1,88). O relato de apnéia obstrutiva foi maior nos homens ( $\mathrm{RP}=2,05$; IC $95 \%$ : 1,67;2,52), nos idosos $(\mathrm{RP}=2,23$; IC 95\%: 1,64;3,03), nos tabagistas ( $\mathrm{RP}=1,60$; IC 95\%: 1,25;2,05) e nos obesos ( $\mathrm{RP}=2,61$; IC 95\%: 1,97;3,47).

CONCLUSÕES: Ronco habitual e apnéia obstrutiva foram sintomas comuns na população estudada. Fatores de risco conhecidos como sexo masculino e idade entre quarta e quinta décadas de vida não são modificáveis. Entretanto, tabagismo, alcoolismo e obesidade também associados aos desfechos, devem ser identificados e tratados na população geral.

DESCRITORES: Síndromes da Apnéia do Sono, epidemiologia. Ronco. Apnéia do Sono Tipo Obstrutiva. Fatores de Risco. Estudos Transversais. 


\section{ABSTRACT}

OBJECTIVE: To estimate the prevalence of habitual snoring and obstructive sleep apnea and their factors associated.

METHODS: Cross-sectional study with a representative sample comprising 3,136 adults ( $\geq 20$ years) living in the city of Pelotas, Southern Brazil, in 2005. A questionnaire was used to collect information on demographic, socioeconomic, behavioral and anthropometric variables and outcomes. Crude and adjusted statistical analysis using Fisher exact test and chi-square test for linear trend and a multivariate analysis through Poisson regression model were carried out, respectively.

RESULTS: The prevalence of habitual snoring was 50.5\% (95\% CI: 48.1;52.8) and obstructive sleep apnea was 9.9\% (95\% CI: 8.7;11.2). After adjustment, the risk of habitual snoring was greater in males (prevalence ratio $[\mathrm{PR}]=1.25,95 \%$ CI: $1.16 ; 1.34)$, elderly people $(\mathrm{PR}=1.62,95 \% \mathrm{CI}: 1.46 ; 1.80)$, current smokers $(\mathrm{PR}=1.15,95 \% \mathrm{CI}: 1.07 ; 1.25)$, alcohol users (PR=1.17, 95\% CI: 1.03;1.31) and obese people ( $\mathrm{PR}=1.71,95 \% \mathrm{CI}: 1.55 ; 1.88)$. The risk of obstructive sleep apnea was greater in males $(\mathrm{PR}=2.05,95 \% \mathrm{CI}: 1.67 ; 2.52)$, elderly people $(\mathrm{PR}=2.23$, 95\% CI: 1.64;3.03), current smokers ( $\mathrm{PR}=1.60,95 \% \mathrm{CI}: 1.25 ; 2.05)$ and obese people (PR=2.61, 95\% CI: 1.97;3.47).

CONCLUSIONS: In the studied population, habitual snoring and obstructive sleep apnea were common symptoms. Well-known risk factors such as male gender and age between 40 and 60 years are not modifiable. However, smoking, alcohol use and obesity need to be identified and treated in general population.

DESCRIPTORS: Sleep Apnea Syndromes, epidemiology. Snoring. Sleep Apnea, Obstructive. Risk Factors. Cross-Sectional Studies.

\section{INTRODUÇÃO}

O ser humano passa aproximadamente um terço de sua vida dormindo. Durante o sono, a vibração do palato e dos tecidos moles adjacentes à faringe produz o ronco, sintoma comum na população geral. ${ }^{19} \mathrm{O}$ ronco sugere a presença de obstrução parcial das vias aéreas superiores e pode ser seguido da apnéia, a qual corresponde à cessação completa do fluxo aéreo na presença de esforço respiratório. ${ }^{1}$

Os distúrbios respiratórios do sono se caracterizam pela presença de episódios repetidos de pausas respiratórias, que levam à fragmentação do sono e à redução da saturação da oxihemoglobina. ${ }^{23}$ Com prevalência de $9 \%$ nas mulheres e $24 \%$ nos homens, ${ }^{23}$ os distúrbios respiratórios do sono representam um espectro contínuo, desde o ronco até a síndrome da apnéia obstrutiva do sono). ${ }^{1,23}$

Estima-se que 2\% a 4\% da população adulta de meia idade seja acometida pela síndrome da apnéia obstrutiva do sono. ${ }^{23}$ Essa síndrome tem sido associada à sonolência diurna excessiva, ${ }^{1}$ a acidentes automobilísticos ${ }^{4} \mathrm{e}$ à morbimortalidade cardiovascular. ${ }^{13}$ Entretanto, o diagnóstico não é realizado em $82 \%$ dos homens e $93 \%$ das mulheres portadoras da síndrome da apnéia obstrutiva do sono moderada à grave. ${ }^{23} \mathrm{~A}$ baixa freqüência do diagnóstico pode ser reflexo da reduzida percepção dos sintomas do sono como um problema ${ }^{9}$ pelo paciente e seus familiares, do difícil acesso aos métodos diagnósticos associados e possivelmente ao treinamento insuficiente em medicina do sono. Uma pesquisa realizada em escolas médicas no Reino Unido estimou que o tempo de ensino específico sobre sono é menor do que 15 minutos no ensino básico e inexistente na formação clínica. ${ }^{18}$

O interesse no ronco habitual e na apnéia obstrutiva é explicado pelas suas relações com a síndrome da apnéia obstrutiva do sono. Sexo masculino, faixa etária entre 40 e 60 anos e obesidade são fatores de risco conhecidos para ronco habitual. ${ }^{2,23,24}$ Tabagismo e alcoolismo também têm sido associados ao ronco. ${ }^{5,7}$ Indivíduos que roncam sofrem freqüentemente de sonolência diurna excessiva ${ }^{24}$ e podem prejudicar a qualidade de vida de seus companheiros. ${ }^{8}$ Mesmo os distúrbios respiratórios do sono leves podem afetar a saúde dos indivíduos, haja vista a associação entre ronco habitual e infarto do miocárdio, acidente vascular cerebral e hipertensão arterial sistêmica. ${ }^{11}$ 
Considerando-se a ausência de estudos que identifiquem a parcela da população em risco de desenvolver a síndrome da apnéia obstrutiva do sono, o presente estudo teve como objetivo estimar a prevalência de ronco habitual e apnéia obstrutiva observada e verificar a associação desses desfechos com características demográficas, socioeconômicas, comportamentais e antropométricas.

\section{MÉTODOS}

Estudo transversal realizado na área urbana de Pelotas, município com aproximadamente 320.000 habitantes, localizado ao sul do Rio Grande do Sul. Por meio de um sistema de consórcio, foi utilizado um único instrumento para coleta de dados de outubro a dezembro de 2005 por pesquisadores com projetos e objetivos diferentes.

Uma amostra populacional representativa foi selecionada por conglomerados em múltiplos estágios. A unidade amostral primária foram os setores censitários do município, delimitados pelo censo realizado em 2000 pelo Instituto Brasileiro de Geografia e Estatística (IBGE). Após estratificação dos 408 setores censitários da cidade, 119 foram sorteados com probabilidade proporcional ao tamanho conforme a renda mensal média do responsável pela família (excluídos os quatro setores desabitados ou comerciais). Em cada setor censitário foram amostrados, de forma sistemática, em média 12 domicílios. Assim, o número final de domicílios elegíveis foi de 1.957 , onde em cada um desses todos os residentes com idade igual ou superior a 20 anos deveriam ser entrevistados.

O tamanho da amostra calculado foi de 2.155 pessoas, considerando-se erro alfa de $5 \%$, poder de $80 \%$, prevalência estimada de apnéia obstrutiva observada de 7\% nos nunca tabagistas e $11 \%$ nos tabagistas, ${ }^{19}$ razão entre não expostos e expostos de 1,1:1 e risco relativo de 1,5. A esse valor foram acrescidos $25 \%$ para perdas, recusas e controle de fatores de confusão, e 15\% para compensar o efeito do delineamento, totalizando 3.017 pessoas.

Para atender todas as necessidades do consórcio de pesquisa, nos 1.957 domicílios, foram considerados elegíveis 3.353 adultos. Ao final do estudo, com uma percentagem total de perdas e recusas de $6,5 \%$, a amostra foi constituída por 3.136 pessoas.

Para operacionalização das variáveis dependentes foram utilizadas perguntas do instrumento do Proyecto Latinoamericano de Investigación en Obstrucción Pulmonar (Projeto Platino), estudo multicêntrico de base populacional realizado na América Latina ${ }^{19}$ que investigou, além de sintomas de enfermidades respiratórias, ronco, sonolência diurna excessiva e apnéias obstrutivas em adultos com 40 anos ou mais. Após tradução e re-tradução das perguntas, ${ }^{19}$ as respostas foram analisadas como variáveis categóricas dicotômi- cas. Ronco - "Já lhe disseram que o(a) senhor(a) ronca todas ou quase todas as noites?"; ronco habitual - relato de ronco há pelo menos 12 meses; apnéia presenciada - "Já lhe disseram que quando o(a) senhor(a) dorme, com freqüência deixa de respirar por alguns momentos?". O desfecho apnéia obstrutiva (dicotômico) foi definido pelo relato concomitante de ronco habitual e apnéia presenciada. Foram coletadas informações sobre o período de latência para iniciar o sono em minutos, e o tempo em anos desde o início do sintoma ronco - ambas variáveis numéricas discretas. Avaliou-se ainda a auto-percepção da qualidade do sono - ruim, regular, bom, muito bom e ótimo.

As variáveis independentes utilizadas foram:

- demográficas - sexo, idade, cor da pele (aferida pela entrevistadora) e situação conjugal (com e sem companheiro);

- $\quad$ socioeconômica - nível socioeconômico, segundo a Associação Brasileira de Empresas de Pesquisa (ABEP), ${ }^{a}$ em cinco categorias (A, B, C, D e E);

- comportamentais - tabagismo: não fumante, ex-fumante ou fumante (um ou mais cigarros por dia, há mais de um mês); alcoolismo (dois ou mais pontos no escore do instrumento CAGE) $;{ }^{6}$ atividade física regular: ativo (exercer atividade física por pelo menos 20 minutos, no mínimo três dias da semana, há pelo menos seis meses) e não ativo; IMC (baseado no peso e na altura referidos pelo entrevistado): indivíduos classificados como desnutrido/normal $\left(<25 \mathrm{~kg} / \mathrm{m}^{2}\right)$, sobrepeso $\left(25-29,9 \mathrm{~kg} / \mathrm{m}^{2}\right)$ e obeso $\left(\geq 30 \mathrm{~kg} / \mathrm{m}^{2}\right){ }^{21}$

Os dados foram digitados duplamente no programa EpiInfo $6.04 \mathrm{~b}$ e a análise estatística realizada com auxílio do pacote estatístico Stata 9. Para controle de qualidade dos dados, aplicou-se uma versão resumida do questionário em $10 \%$ dos entrevistados. A concordância entre as respostas foi avaliada pelo coeficiente kappa $(0,72 \pm 0,03)$ para a variável escolaridade e de $(0,83 \pm 0,04)$ para tabagismo.

Após descrição da amostra, realizou-se análise bruta das variáveis dependentes e sua associação com as independentes, por meio do teste exato de Fisher para associação, qui-quadrado para tendência linear e análise de variância (ANOVA) com teste de Bonferroni. As análises de razões de prevalência brutas e ajustadas por níveis foram feitas a partir de um modelo hierarquizado. Este modelo foi composto por variáveis demográficas no nível mais distal, pela variável socioeconômica no segundo nível, no terceiro por variáveis comportamentais e as antropométricas no nível mais proximal. Além disso, foi realizada análise estratificada por sexo. Para cada nível da análise, realizou-se regressão de Poisson, sempre

a Associação Brasileira de Empresas de Pesquisa. Critério brasileiro de classificação econômica. São Paulo; 2003. 
se considerando o efeito do delineamento amostral, utilizando o comando svy. O nível de significância utilizado para associações entre variáveis independentes e variáveis dependentes foi de $5 \%$. Por eliminação retrógrada, decidiu-se manter as variáveis com valor $\mathrm{p} \leq 0,20$ para controle de fatores de confusão.

O estudo foi aprovado pelo Comitê de Ética em Pesquisa da Universidade Federal de Pelotas, e todos os entrevistados assinaram um termo de consentimento livre e esclarecido antes de responder ao questionário.

\section{RESULTADOS}

A Tabela 1 apresenta uma análise dos desfechos estudados e sua associação com fatores demográficos, socioeconômicos, tabagismo, alcoolismo, atividade física e IMC. A média de idade dos entrevistados foi de 44,1 ( $\pm 16,4 \mathrm{dp})$ anos, a maioria (56\%) era do sexo feminino, de cor branca $(84 \%)$. Com relação à situação conjugal, $37,2 \%$ dos entrevistados relataram não ter companheiro(a), desses, 65\% eram mulheres. Quanto ao nível socioeconômico, cerca de $40 \%$ pertenciam à

Tabela 1. Descrição da amostra e prevalência de ronco habitual e apnéia obstrutiva em adultos. Pelotas, RS, 2005. (N=3.136)

\begin{tabular}{|c|c|c|c|c|c|c|}
\hline \multirow{2}{*}{ Variável } & \multirow{2}{*}{$\mathrm{N}$} & \multirow{2}{*}{$\%$} & \multicolumn{2}{|c|}{ Ronco habitual } & \multicolumn{2}{|c|}{ Apnéia obstrutiva } \\
\hline & & & $\%($ IC 95\%) & $\mathrm{p}$ & $\%($ IC 95\%) & $\mathrm{p}$ \\
\hline Sexo* & & & & $<0,001$ & & $<0,001$ \\
\hline Feminino & 1.758 & 56,1 & $45,2(42,3 ; 48,2)$ & & $6,8(5,6 ; 8,0)$ & \\
\hline Masculino & 1.378 & 43,9 & $57,0(54,0 ; 60,0)$ & & $13,9(11,7 ; 16,2)$ & \\
\hline Idade $\left(\right.$ anos) ${ }^{* *}$ & & & & $<0,001$ & & $<0,001$ \\
\hline $20-39$ & 1.358 & 43,3 & $37,0(34,0 ; 40,1)$ & & $6,2(4,8 ; 7,6)$ & \\
\hline $40-59$ & 1.181 & 37,7 & $61,4(58,0 ; 64,8)$ & & $12,6(10,6 ; 14,6)$ & \\
\hline$\geq 60$ & 597 & 19,0 & $59,2(54,8 ; 63,6)$ & & $13,2(10,2 ; 16,2)$ & \\
\hline Cor da pele* & & & & 0,3 & & 0,4 \\
\hline Não branca & 502 & 16,0 & $48,4(43,6 ; 53,2)$ & & $8,8(5,7 ; 11,9)$ & \\
\hline Branca & 2.634 & 84,0 & $50,8(48,3 ; 53,4)$ & & $10,2(8,7 ; 10,6)$ & \\
\hline Situação conjugal* & & & & $<0,001$ & & 0,001 \\
\hline Sem companheiro & 1.168 & 37,2 & $42,2(38,9 ; 45,5)$ & & $7,6(6,0 ; 9,2)$ & \\
\hline Com companheiro & 1.968 & 62,8 & $55,4(52,5 ; 58,2)$ & & $11,3(9,6 ; 13,0)$ & \\
\hline Nível socioeconômico* & & & & 0,05 & & 0,3 \\
\hline A & 161 & 5,1 & $47,4(38,8 ; 56,1)$ & & $12,4(7,5 ; 17,4)$ & \\
\hline B & 824 & 26,5 & $53,4(49,6 ; 57,2)$ & & $9,8(7,8 ; 11,8)$ & \\
\hline $\mathrm{C}$ & 1.295 & 41,6 & $51,5(48,2 ; 54,8)$ & & $10,2(8,3 ; 12,1)$ & \\
\hline $\mathrm{D}$ & 762 & 24,5 & $48,1(44,4 ; 51,8)$ & & $10,0(7,5 ; 12,5)$ & \\
\hline $\mathrm{E}$ & 73 & 2,3 & $36,8(23,1 ; 50,4)$ & & $2,7(-1,2 ; 6,7)$ & \\
\hline Tabagismo** & & & & $<0,001$ & & $<0,001$ \\
\hline Não fumante & 1.634 & 52,1 & $44,9(42,0 ; 47,7)$ & & $6,9(5,5 ; 8,2)$ & \\
\hline Ex-fumante & 664 & 21,2 & $56,5(51,8 ; 61,2)$ & & $13,7(10,9 ; 16,5)$ & \\
\hline Fumante & 838 & 26,7 & $56,6(53,0 ; 60,1)$ & & $13,0(10,4 ; 15,6)$ & \\
\hline Alcoolismo* & & & & $<0,001$ & & 0,001 \\
\hline Não & 2.959 & 94,4 & $49,6(47,2 ; 52,0)$ & & $9,5(8,2 ; 10,8)$ & \\
\hline Sim & 177 & 5,6 & $64,5(57,4 ; 71,6)$ & & $18,1(12,4 ; 23,7)$ & \\
\hline Atividade física regular* & & & & 0,8 & & 0,8 \\
\hline Ativo & 754 & 24,1 & $50,0(46,1 ; 53,9)$ & & $10,2(8,1 ; 12,3)$ & \\
\hline Não ativo & 2.381 & 75,9 & $50,6(48,0 ; 53,2)$ & & $9,9(8,4 ; 11,3)$ & \\
\hline $\operatorname{IMC}\left(\mathrm{kg} / \mathrm{m}^{2}\right)^{* *}$ & & & & $<0,001$ & & $<0,001$ \\
\hline$<25$ & 1.468 & 48,4 & $38,1(35,0 ; 41,1)$ & & $6,6(5,2 ; 8,0)$ & \\
\hline $25-29,9$ & 1.092 & 36,0 & $58,8(55,6 ; 62,1)$ & & $10,7(8,7 ; 12,7)$ & \\
\hline$\geq 30$ & 471 & 15,6 & $70,7(65,8 ; 75,5)$ & & $18,7(15,0 ; 22,4)$ & \\
\hline
\end{tabular}

* Teste do qui-quadrado exato de Fisher

** Teste do qui-quadrado de tendência linear

Nota: A variável com maior número de valores ignorados foi IMC (3,4\%). 
classe C. Pouco menos da metade dos entrevistados era fumante ou ex-fumante. A maioria dos não fumantes era do sexo feminino (65\%); alcoolismo foi relatado por $5 \% ; 75,9 \%$ foi considerado não ativo; em relação ao IMC, $48,4 \%$ eram normais ou desnutridos.

A média de horas de sono foi de 7,5 horas $( \pm 1,8 \mathrm{dp})$, tendo sido menor nos homens $(7,4 \mathrm{~h} \pm 1,7 \mathrm{dp})$ do que nas mulheres $(7,6 h \pm 1,8 \mathrm{dp})(\mathrm{p}<0,001)$. A mediana do período de latência para iniciar o sono foi de $15 \mathrm{minu}-$ tos. Aproximadamente $26 \%$ das pessoas perceberam o sono como regular ou ruim.

A prevalência de ronco habitual foi de 50,5\% (IC 95\%: 48,$1 ; 52,8$ ) e a de apnéia obstrutiva de $9,9 \%$ (IC 95\%: $8,7 ; 11,2)$. O relato de ronco habitual foi mais freqüente nos homens $(60 \%)$, tendo atingido a mesma proporção nos entrevistados com idade entre 40 e 59 anos. Pessoas sem companheiro relataram-no menos freqüentemente do que aquelas com companheiro. Houve tendência de aumento da prevalência com o aumento do nível socioeconômico (36,6\% no nível E a 47,3\% no nível A). Em comparação com os não tabagistas e não alcoolistas, os tabagistas atuais, os ex-tabagistas e os alcoolistas, apresentaram maiores prevalências $(56,5 \%, 56,6 \%$ e $64 \%$, respectivamente). Os indivíduos obesos apresentaram prevalências maiores do que os não obesos, com ocorrência de mais de $70 \%$.

Para o desfecho apnéia obstrutiva, as mulheres apresentaram uma prevalência menor do que a encontrada nos homens ( $7 \%$ vs. $14 \%$, respectivamente). Constatou-se tendência de aumento da prevalência de apnéia obstrutiva com o avançar da idade. A ocorrência foi maior nas pessoas com companheiro $(11,3 \%)$ do que nas sem companheiro (7,6\%). Aproximadamente um quinto dos alcoolistas, $15 \%$ dos tabagistas e $20 \%$ dos indivíduos obesos relataram apnéia obstrutiva.

A Tabela 2 mostra razões de prevalência para o relato de ronco habitual, com os respectivos intervalos de confiança (IC 95\%) e valores p nas análises bruta e ajustada. As variáveis estudadas foram divididas em níveis hierárquicos. Após ajuste para fatores de confusão, os homens apresentaram prevalência quase $25 \%$ maior do que as mulheres. A ocorrência desse desfecho foi 1,6 vezes maior nas pessoas com idade maior ou igual a 40 anos do que nas com idade entre 20 e 39 anos. Indivíduos com companheiro apresentaram 1,2 vezes mais ocorrência do que os sem companheiro. Fumantes e alcoolistas apresentaram prevalências 15\% e $17 \%$ maior do que não fumantes e não alcoolistas, respectivamente. A prevalência em obesos foi 1,7 vez maior do que os com IMC normal $(\mathrm{p}<0,001)$.

A Tabela 3 mostra razões de prevalência para o relato de apnéia obstrutiva, com respectivos IC 95\% e valores de $\mathrm{p}$ das análises bruta e ajustada. Na análise ajustada, este desfecho foi duas vezes mais freqüentes nos homens do que nas mulheres. Observou-se aumento da sua prevalência com o avançar da idade. Idosos apresentaram prevalência 2,2 vezes maior do que as pessoas com idade entre 20 e 39 anos. Após ajuste para sexo e idade, "ter companheiro" perdeu associação com o desfecho. Sua ocorrência foi 1,6 vezes maior nos tabagistas do que nos não tabagistas. Alcoolismo perdeu associação após o ajuste para os fatores de confusão. Obesos apresentaram prevalência 2,6 vezes maior do que indivíduos com IMC normal.

Assim como para o relato de ronco habitual, cor da pele, atividade física regular e nível socioeconômico não estiveram associados ao relato de apnéia obstrutiva.

$\mathrm{Na}$ análise estratificada por sexo (Tabela 4), foram observadas algumas diferenças nas medidas de efeito após ajuste para fatores de confusão. A idade modificou a associação entre as variáveis sexo e ronco habitual ( $p$ interação $<0,001)$. As razões de prevalência $(\mathrm{RP})$ para ronco habitual entre idosos e pessoas com idade entre 20 e 39 anos mostraram que a influência do avanço da idade foi maior nas mulheres do que nos homens $(\mathrm{RP}=2,05$ e $\mathrm{RP}=1,13$, respectivamente). A associação entre ronco habitual e situação conjugal permaneceu significativa apenas no sexo masculino. Homens com companheira apresentaram aumento nas prevalências de ronco habitual e apnéia obstrutiva, em relação aos sem companheira (64\% e de $100 \%$, respectivamente). Observou-se que, nos homens, quanto menor o nível socioeconômico, menor foi a ocorrência de ronco habitual e apnéia obstrutiva. Após ajuste para fatores de confusão, mulheres alcoolistas apresentaram prevalência de apnéia obstrutiva 3,45 (IC 95\%: 1,90; 6,29) vezes maior do que a das não alcoolistas $(\mathrm{p}<0,001)$, o que não foi verificado na análise geral.

Os efeitos de delineamento encontrados foram 1,68 para o desfecho ronco habitual e 1,46 para apnéia obstrutiva.

\section{DISCUSSÃO}

No Brasil, sintomas dos distúrbios respiratórios do sono têm sido estudados em populações específicas. ${ }^{4,12}$ O presente estudo é o primeiro estudo transversal de base populacional realizado no Brasil com o objetivo de investigar concomitantemente ronco habitual e apnéia obstrutiva observada. Considerando-se que estes sintomas são freqüentes e geralmente presentes ao longo da vida, o estudo transversal, apesar de não permitir inferências causais, é o delineamento ideal para determinação de prevalências e associações.

Apesar da baixa taxa de perdas e recusas (6,5\%), algumas limitações do presente estudo devem ser apontadas. Em laboratórios de pesquisa, o ronco pode ser gravado com auxílio de microfones colocados sobre o esterno ou região do mento, digitalmente processado e analisado. ${ }^{8}$ 
Tabela 2. Análise bruta e ajustada das razões de prevalência e intervalos de confiança (95\%) para ronco habitual em adultos segundo características demográficas, socioeconômicas, comportamentais e antropométricas. Pelotas, RS, 2005. ( $N=$ 3.136)

\begin{tabular}{|c|c|c|c|c|c|}
\hline \multirow{2}{*}{ Nível* } & \multirow{2}{*}{ Variável } & \multicolumn{2}{|c|}{ Análise bruta } & \multicolumn{2}{|c|}{ Análise ajustada } \\
\hline & & RP (IC 95\%) & $\mathrm{p}$ & RP (IC 95\%) & $\mathrm{p}$ \\
\hline \multirow[t]{3}{*}{1} & Sexo** & & $<0,001$ & & $<0,001$ \\
\hline & Feminino & 1,00 & & 1,00 & \\
\hline & Masculino & $1,26(1,17 ; 1,35)$ & & $1,25(1,16 ; 1,34)$ & \\
\hline \multirow[t]{4}{*}{1} & Idade (anos) $)^{* * *}$ & & $<0,001$ & & $<0,001$ \\
\hline & $20-39$ & 1,00 & & 1,00 & \\
\hline & $40-59$ & $1,66(1,51 ; 1,82)$ & & $1,63(1,49 ; 1,80)$ & \\
\hline & $\geq 60$ & $1,60(1,44 ; 1,77)$ & & $1,62(1,46 ; 1,80)$ & \\
\hline \multirow[t]{3}{*}{1} & Cor da pele** & & 0,3 & & 0,6 \\
\hline & Não branca & 1,00 & & 1,00 & \\
\hline & Branca & $1,05(0,95 ; 1,17)$ & & $1,03(0,93 ; 1,14)$ & \\
\hline \multirow[t]{3}{*}{1} & Situação conjugal** & & $<0,001$ & & $<0,001$ \\
\hline & Sem companheiro & 1,00 & & 1,00 & \\
\hline & Com companheiro & $1,31(1,20 ; 1,43)$ & & $1,21(1,12 ; 1,32)$ & \\
\hline \multirow[t]{6}{*}{2} & Nível socioeconômico*** & & 0,06 & & 0,4 \\
\hline & $A$ & 1,00 & & 1,00 & \\
\hline & B & $1,13(0,93 ; 1,36)$ & & $1,17(0,98 ; 1,40)$ & \\
\hline & $\mathrm{C}$ & $1,09(0,91 ; 1,29)$ & & $1,17(0,99 ; 1,37)$ & \\
\hline & $\mathrm{D}$ & $1,01(0,84 ; 1,23)$ & & $1,10(0,91 ; 1,32)$ & \\
\hline & $\mathrm{E}$ & $0,78(0,52 ; 1,16)$ & & $0,86(0,57 ; 1,29)$ & \\
\hline \multirow[t]{4}{*}{3} & Tabagismo*** & & $<0,001$ & & 0,001 \\
\hline & Não fumante & 1,00 & & 1,00 & \\
\hline & Ex-fumante & $1,26(1,14 ; 1,39)$ & & $1,05(0,95 ; 1,16)$ & \\
\hline & Fumante & $1,26(1,17 ; 1,36)$ & & $1,15(1,07 ; 1,25)$ & \\
\hline \multirow[t]{3}{*}{3} & Alcoolismo** & & $<0,001$ & & 0,01 \\
\hline & Não & 1,00 & & 1,00 & \\
\hline & Sim & $1,30(1,16 ; 1,46)$ & & $1,17(1,03 ; 1,31)$ & \\
\hline \multirow[t]{3}{*}{3} & Atividade física regular** & & 0,8 & & 0,7 \\
\hline & Ativo & 1,00 & & 1,00 & \\
\hline & Não ativo & $1,01(0,93 ; 1,10)$ & & $1,02(0,94 ; 1,10)$ & \\
\hline \multirow[t]{4}{*}{4} & $\operatorname{IMC}\left(\mathrm{kg} / \mathrm{m}^{2}\right)^{* * *}$ & & $<0,001$ & & $<0,001$ \\
\hline & $<25$ & 1,00 & & 1,00 & \\
\hline & $25-29,9$ & $1,54(1,42 ; 1,68)$ & & $1,44(1,32 ; 1,56)$ & \\
\hline & $\geq 30$ & $1,86(1,68 ; 2,05)$ & & $1,71(1,55 ; 1,88)$ & \\
\hline
\end{tabular}

\footnotetext{
* Níveis hierárquicos de determinação: ajuste para variáveis do mesmo nível ou nível superior, se valor $\mathrm{p} \leq 0,20$

** Teste de Wald de associação

*** Teste de Wald de tendência linear

RP: Razões de prevalência
}

Entretanto, como os sons captados pelo microfone ou percebidos pelo técnico do laboratório podem ser ruídos do ambiente, tosse ou mobilização do microfone, esse método não é considerado isento de limitações. ${ }^{9} \mathrm{~A}$ polissonografia noturna é o método de escolha e mais adequado para definição de ronco e apnéia, pois tem a capacidade de identificar e quantificar o número e a duração dos eventos respiratórios. ${ }^{1}$ Porém, o alto custo e as dificuldades operacionais tornam sua aplicação inviável em estudos populacionais. Considerando que a percepção do ronco é essencialmente uma impressão subjetiva do ouvinte, pesquisadores têm utilizado questionários, com desfechos dicotômicos ou escalas (de três, quatro ou cinco pontos), para investigar o sintoma ronco. ${ }^{2,8,19} \mathrm{Na}$ maioria desses estudos, para que o ronco seja considerado "habitual" é necessário que esteja presente todos ou quase todos os dias da semana. 
Tabela 3. Análise bruta e ajustada das razões de prevalência e intervalos de confiança (95\%) para apnéia obstrutiva em adultos segundo características demográficas, socioeconômicas, comportamentais e antropométricas. Pelotas, RS, 2005. (N = 3.136)

\begin{tabular}{|c|c|c|c|c|c|}
\hline \multirow{2}{*}{ Nível* } & \multirow{2}{*}{ Variável } & \multicolumn{2}{|c|}{ Análise bruta } & \multicolumn{2}{|c|}{ Análise ajustada } \\
\hline & & RP (IC 95\%) & $\mathrm{p}$ & RP (IC 95\%) & $\mathrm{p}$ \\
\hline \multirow[t]{3}{*}{1} & Sexo** & & $<0,001$ & & $<0,001$ \\
\hline & Feminino & 1,00 & & 1,00 & \\
\hline & Masculino & $2,04(1,65 ; 2,53)$ & & $2,05(1,67 ; 2,52)$ & \\
\hline \multirow[t]{4}{*}{1} & Idade (anos) $)^{* * *}$ & & $<0,001$ & & $<0,001$ \\
\hline & $20-39$ & 1,00 & & 1,00 & \\
\hline & $40-59$ & $2,04(1,61 ; 2,59)$ & & $2,04(1,61 ; 2,60)$ & \\
\hline & $\geq 60$ & $2,14(1,57 ; 2,92)$ & & $2,23(1,64 ; 3,03)$ & \\
\hline \multirow[t]{3}{*}{1} & Cor da pele** & & 0,4 & & 0,5 \\
\hline & Não branca & 1,00 & & 1,00 & \\
\hline & Branca & $1,16(0,79 ; 1,71)$ & & $1,13(0,76 ; 1,67)$ & \\
\hline \multirow[t]{3}{*}{1} & Situação conjugal** & & 0,002 & & 0,1 \\
\hline & Sem companheiro & 1,00 & & 1,00 & \\
\hline & Com companheiro & $1,49(1,16 ; 1,90)$ & & $1,22(0,95 ; 1,56)$ & \\
\hline \multirow[t]{6}{*}{2} & Nível socioeconômico*** & & 0,2 & & 0,6 \\
\hline & A & 1,00 & & 1,00 & \\
\hline & B & $0,79(0,51 ; 1,23)$ & & $0,84(0,55 ; 1,29)$ & \\
\hline & $\mathrm{C}$ & $0,82(0,54 ; 1,26)$ & & $0,92(0,61 ; 1,37)$ & \\
\hline & $\mathrm{D}$ & $0,80(0,51 ; 1,27)$ & & $0,90(0,57 ; 1,42)$ & \\
\hline & $\mathrm{E}$ & $0,22(0,05 ; 0,95)$ & & $0,26(0,06 ; 1,13)$ & \\
\hline \multirow[t]{4}{*}{3} & Tabagismo*** & & $<0,001$ & & $<0,001$ \\
\hline & Não fumante & 1,00 & & 1,00 & \\
\hline & Ex-fumante & $2,00(1,52 ; 2,64)$ & & $1,42(1,06 ; 1,90)$ & \\
\hline & Fumante & $1,90(1,48 ; 2,44)$ & & $1,60(1,25 ; 2,05)$ & \\
\hline \multirow[t]{3}{*}{3} & Alcoolismo** & & $<0,001$ & & 0,07 \\
\hline & Não & 1,00 & & 1,00 & \\
\hline & Sim & $1,91(1,37 ; 2,67)$ & & $1,38(0,97 ; 1,96)$ & \\
\hline \multirow[t]{3}{*}{3} & Atividade física regular** & & 0,8 & & 0,9 \\
\hline & Ativo & 1,00 & & 1,00 & \\
\hline & Não ativo & $0,97(0,77 ; 1,22)$ & & $1,00(0,80 ; 1,25)$ & \\
\hline \multirow[t]{4}{*}{4} & $\operatorname{IMC}\left(\mathrm{kg} / \mathrm{m}^{2}\right)^{* * *}$ & & $<0,001$ & & $<0,001$ \\
\hline & $<25$ & 1,00 & & 1,00 & \\
\hline & $25-29,9$ & $1,62(1,29 ; 2,05)$ & & $1,47(1,17 ; 1,83)$ & \\
\hline & $\geq 30$ & $2,83(1,13 ; 3,76)$ & & $2,61(1,97 ; 3,47)$ & \\
\hline
\end{tabular}

* Níveis hierárquicos de determinação: ajuste para variáveis do mesmo nível ou nível superior, se valor $p \leq 0,20$

** Teste de Wald de associação

*** Teste de Wald de tendência linear

Outra limitação foi a não realização da aferição da circunferência cervical. Essa medida está relacionada à obesidade e tem alta correlação com a síndrome da apnéia obstrutiva do sono. ${ }^{10} \mathrm{O}$ IMC foi calculado com base no peso e altura referido, o que pode trazer implicações com respeito à precisão das medidas. Existem, no entanto, evidências na literatura de que, em geral, o peso referido pela população adulta tem alta correlação com o peso medido, tornando improvável essa fonte de erro. ${ }^{17}$ Além disso, não foi investigada a utilização de medicamentos indutores do sono, uma vez que o uso dessas medicações e do álcool está associado ao relato de ronco. ${ }^{16}$

A utilização do instrumento do Projeto Platino com definição dicotômica do desfecho possibilitou maior comparabilidade entre os resultados do Platino e do presente estudo, principalmente no que se refere às prevalências. 
Tabela 4. Análise ajustada, estratificada por sexo, das razões de prevalência e intervalos de confiança (95\%) para ronco habitual e apnéia obstrutiva em adultos segundo características demográficas, socioeconômicas, comportamentais e antropométricas. Pelotas, RS, 2005. ( $\mathrm{N}=3.136)$

\begin{tabular}{|c|c|c|c|c|c|c|c|c|c|}
\hline \multirow{3}{*}{ Nível* } & \multirow{3}{*}{ Variável } & \multicolumn{4}{|c|}{ Ronco habitual } & \multicolumn{4}{|c|}{ Apnéia obstrutiva } \\
\hline & & \multicolumn{2}{|c|}{ Mulher } & \multicolumn{2}{|c|}{ Homem } & \multicolumn{2}{|c|}{ Mulher } & \multicolumn{2}{|c|}{ Homem } \\
\hline & & $\begin{array}{c}\mathrm{RP} \\
(\mathrm{IC} 95 \%)\end{array}$ & $\mathrm{p}$ & $\begin{array}{c}\text { RP } \\
\text { (IC 95\%) }\end{array}$ & $\mathrm{p}$ & $\begin{array}{c}\mathrm{RP} \\
\text { (IC 95\%) }\end{array}$ & $\mathrm{p}$ & $\begin{array}{c}\mathrm{RP} \\
\text { (IC 95\%) }\end{array}$ & $\mathrm{p}$ \\
\hline \multirow[t]{4}{*}{1} & Idade (anos) $)^{* *}$ & & $<0,001$ & & 0,001 & & $<0,001$ & & $<0,001$ \\
\hline & $20-39$ & 1,00 & & 1,00 & & 1,00 & & 1,00 & \\
\hline & $40-59$ & $\begin{array}{c}2,01 \\
(1,74 ; 2,33)\end{array}$ & & $\begin{array}{c}1,30 \\
(1,17 ; 1,43)\end{array}$ & & $\begin{array}{c}2,64 \\
(1,75 ; 4,00)\end{array}$ & & $\begin{array}{c}1,62 \\
(1,19 ; 2,21)\end{array}$ & \\
\hline & $\geq 60$ & $\begin{array}{c}2,05 \\
(1,76 ; 2,40)\end{array}$ & & $\begin{array}{c}1,17 \\
(1,03 ; 1,34)\end{array}$ & & $\begin{array}{c}2,50 \\
(1,46 ; 4,30)\end{array}$ & & $\begin{array}{c}1,82 \\
(1,28 ; 2,60)\end{array}$ & \\
\hline \multirow[t]{3}{*}{1} & Cor da pele*** & & 0,9 & & 0,3 & & 0,7 & & 0,6 \\
\hline & Não branca & 1,00 & & 1,00 & & 1,00 & & 1,00 & \\
\hline & Branca & $\begin{array}{c}1,00 \\
(0,86 ; 1,16)\end{array}$ & & $\begin{array}{c}1,07 \\
(0,94 ; 1,22)\end{array}$ & & $\begin{array}{c}1,11 \\
(0,65 ; 1,88)\end{array}$ & & $\begin{array}{c}1,15 \\
(0,73 ; 1,81)\end{array}$ & \\
\hline \multirow[t]{3}{*}{1} & Situação conjugal ${ }^{* * *}$ & & 0,3 & & $<0,001$ & & 0,2 & & 0,001 \\
\hline & Sem companheiro & 1,00 & & 1,00 & & 1,00 & & 1,00 & \\
\hline & Com companheiro & $\begin{array}{c}1,06 \\
(0,95 ; 1,17)\end{array}$ & & $\begin{array}{c}1,64 \\
(1,43 ; 1,88)\end{array}$ & & $\begin{array}{c}0,79 \\
(0,55 ; 1,12)\end{array}$ & & $\begin{array}{c}2,01 \\
(1,33 ; 3,04)\end{array}$ & \\
\hline \multirow[t]{6}{*}{2} & Nível socioeconômico** & & 0,9 & & 0,02 & & 0,2 & & 0,04 \\
\hline & A & 1,00 & & 1,00 & & 1,00 & & 1,00 & \\
\hline & B & $\begin{array}{c}1,44 \\
(1,01 ; 2,07)\end{array}$ & & $\begin{array}{c}0,99 \\
(0,85 ; 1,14)\end{array}$ & & $\begin{array}{c}0,89 \\
(0,35 ; 2,22)\end{array}$ & & $\begin{array}{c}0,81 \\
(0,49 ; 1,35)\end{array}$ & \\
\hline & $\mathrm{C}$ & $\begin{array}{c}1,55 \\
(1,10 ; 2,17)\end{array}$ & & $\begin{array}{c}0,89 \\
(0,78 ; 1,03)\end{array}$ & & $\begin{array}{c}1,23 \\
(0,53 ; 2,88)\end{array}$ & & $\begin{array}{c}0,77 \\
(0,47 ; 1,27)\end{array}$ & \\
\hline & $\mathrm{D}$ & $\begin{array}{c}1,38 \\
(0,97 ; 1,97)\end{array}$ & & $\begin{array}{c}0,89 \\
(0,76 ; 1,04)\end{array}$ & & $\begin{array}{c}1,51 \\
(0,60 ; 3,81)\end{array}$ & & $\begin{array}{c}0,62 \\
(0,35 ; 1,12)\end{array}$ & \\
\hline & $\mathrm{E}$ & $\begin{array}{c}0,97 \\
(0,53 ; 1,78)\end{array}$ & & $\begin{array}{c}0,78 \\
(0,52 ; 1,18)\end{array}$ & & $\begin{array}{c}0,41 \\
(0,05 ; 3,44)\end{array}$ & & $\begin{array}{c}0,20 \\
(0,03 ; 1,55)\end{array}$ & \\
\hline \multirow[t]{4}{*}{3} & Tabagismo** & & 0,04 & & 0,001 & & 0,2 & & $<0,001$ \\
\hline & Não fumante & 1,00 & & 1,00 & & 1,00 & & 1,00 & \\
\hline & Ex-fumante & $\begin{array}{c}1,01 \\
(0,87 ; 1,17)\end{array}$ & & $\begin{array}{c}1,15 \\
(1,02 ; 1,29)\end{array}$ & & $\begin{array}{c}1,26 \\
(0,75 ; 2,11)\end{array}$ & & $\begin{array}{c}1,64 \\
(1,16 ; 2,32)\end{array}$ & \\
\hline & Fumante & $\begin{array}{c}1,15 \\
(1,01 ; 1,31)\end{array}$ & & $\begin{array}{c}1,19 \\
(1,07 ; 1,33)\end{array}$ & & $\begin{array}{c}1,31 \\
(0,88 ; 1,96)\end{array}$ & & $\begin{array}{c}1,93 \\
(1,38 ; 2,71)\end{array}$ & \\
\hline \multirow[t]{3}{*}{3} & Alcoolismo*** & & 0,009 & & 0,02 & & $<0,001$ & & 0,5 \\
\hline & Não & 1,00 & & 1,00 & & 1,00 & & 1,00 & \\
\hline & Sim & $\begin{array}{c}1,38 \\
(1,09 ; 1,75)\end{array}$ & & $\begin{array}{c}1,14 \\
(1,01 ; 1,29)\end{array}$ & & $\begin{array}{c}3,45 \\
(1,90 ; 6,29)\end{array}$ & & $\begin{array}{c}1,15 \\
(0,76 ; 1,74)\end{array}$ & \\
\hline \multirow[t]{3}{*}{3} & $\begin{array}{l}\text { Atividade física } \\
\text { regular*** }\end{array}$ & & 0,4 & & 0,1 & & 0,5 & & 0,5 \\
\hline & Ativo & 1,00 & & 1,00 & & 1,00 & & 1,00 & \\
\hline & Não ativo & $\begin{array}{c}0,95 \\
(0,85 ; 1,08)\end{array}$ & & $\begin{array}{c}1,09 \\
(0,97 ; 1,22)\end{array}$ & & $\begin{array}{c}0,87 \\
(0,56 ; 1,35)\end{array}$ & & $\begin{array}{c}1,09 \\
(0,83 ; 1,44)\end{array}$ & \\
\hline \multirow[t]{4}{*}{4} & IMC $\left(\mathrm{kg} / \mathrm{m}^{2}\right)^{* *}$ & & $<0,001$ & & $<0,001$ & & 0,001 & & $<0,001$ \\
\hline & $<25$ & 1,00 & & 1,00 & & 1,00 & & 1,00 & \\
\hline & $25-29,9$ & $\begin{array}{c}1,39 \\
(1,24 ; 1,55)\end{array}$ & & $\begin{array}{c}1,44 \\
(1,29 ; 1,61)\end{array}$ & & $\begin{array}{c}1,01 \\
(0,73 ; 1,42)\end{array}$ & & $\begin{array}{c}1,72 \\
(1,28 ; 2,31)\end{array}$ & \\
\hline & $\geq 30$ & $\begin{array}{c}1,72 \\
(1,51 ; 1,96)\end{array}$ & & $\begin{array}{c}1,59 \\
(1,40 ; 1,80)\end{array}$ & & $\begin{array}{c}2,20 \\
(1,44 ; 3,34)\end{array}$ & & $\begin{array}{c}2,67 \\
(1,84 ; 3,89)\end{array}$ & \\
\hline
\end{tabular}

\footnotetext{
* Níveis hierárquicos de determinação: ajuste para variáveis do mesmo nível ou nível superior, se valor $p \leq 0,20$

** Teste de Wald de tendência linear

*** Teste de Wald de associação
} 
A ocorrência de ronco habitual em indivíduos com mais de 39 anos atingiu 60,7\% (IC 95\%: 57,9;63,4), percentagem semelhante à encontrada em Montevidéu $(59,4 \%)$, maior do que na Cidade do México $(54,8 \%)$, e menor do que em Santiago do Chile $(66,3 \%) .{ }^{19} \mathrm{~A}$ prevalência de apnéia obstrutiva $(9,9 \%)$ foi próxima da encontrada em Santiago do Chile (11,0\%) e Montevidéu $(8,9 \%)$, porém duas vezes maior do que a observada na Cidade do México (4,9\%). ${ }^{19}$

Estudos mostram que o ronco é mais comum nos homens e nas pessoas obesas, aumentando sua prevalência com o avançar da idade..$^{2,14,19,23}$ Alterações na anatomia e fisiologia das vias aéreas relacionadas ao sexo masculino e à obesidade podem ser a razão das diferenças observadas entre os sexos. Em obesos, a resistência ao fluxo aéreo é maior e potencializada pelo depósito de tecido adiposo em torno da via aérea, além de alterações na função dos músculos respiratórios. ${ }^{3}$ Por outro lado, o sub-relato dos sintomas pelas mulheres e a menor percepção e valorização, por parte dos homens, dos sintomas apresentados pelas suas companheiras, pode ser um fator importante a ser considerado. ${ }^{15} \mathrm{Em}$ relação à idade, observa-se distribuição em forma de "U" invertido, atingindo-se as maiores prevalências nos indivíduos com idade entre 40 e 59 anos. Por meio de um teste de interação, verificou-se que a associação de ronco habitual com o avanço da idade é diferente entre os sexos. No estrato de pessoas com mais de 59 anos, não houve diferença entre as prevalências de ronco habitual: 57,7\% nas mulheres (IC 95\%: 51,6; 63,9\%) e $61,3 \%$ nos homens (IC 95\%: 55,3; 67,4). Esse efeito pode ser explicado em parte pela menor proteção dos hormônios femininos após a menopausa. ${ }^{22}$

É plausível que pessoas com companheiro fixo tenham maior chance de ter seus sintomas percebidos como um problema. Em 1997, Ohayon et al ${ }^{14}$ encontraram maior ocorrência de ronco na categoria de homens casados, com odds ratio de 1,9 (IC 95\%: 1,$7 ; 2,1$ ) em relação aos sem companheira. No presente estudo, esse risco foi 1,4 vez maior (IC 95\%: 1,29; 1,53) para o relato de ronco habitual, e 2,67 vezes maior (IC 95\%: 2,05; $3,50)$ para o relato de apnéia obstrutiva. Entretanto, esse achado pode ter sido reflexo do predomínio do sexo feminino no grupo sem companheiro (65\%). Ao se controlar o efeito da situação conjugal, por meio de análise estratificada, não houve diferença entre os sexos entre as pessoas sem companheiro, tanto para o relato de ronco habitual $(\mathrm{p}=0,8)$, quanto para o relato de apnéia obstrutiva $(p=0,8)$. Em virtude da definição utilizada para o desfecho, é possível que entrevistados sem companheiro tenham sido classificados como não roncadores devido à ausência de um "observador".

A associação encontrada entre ronco habitual e apnéia obstrutiva e o nível socioeconômico nos homens, com maiores prevalências de ambos os desfechos nas ca- madas sociais mais elevadas, pode ser explicada pela relação entre obesidade e nível socioeconômico. A prevalência de obesidade aumenta com o aumento do nível socioeconômico nos homens e o inverso ocorre nas mulheres. Ao se ajustar para o efeito do IMC, as associações entre sexo masculino e os relatos de ronco habitual e de apnéia obstrutiva tornaram-se não significativas.

O estreitamento da via aérea durante o sono desencadeia uma seqüência de eventos, seguida pela redução do fluxo aéreo, vibração dos tecidos moles da faringe, palato mole e úvula. Isso resulta na produção do ronco e pode determinar a interrupção total do fluxo aéreo e conseqüente apnéia. ${ }^{3}$ Logo, pode-se considerar o ronco como um elo perceptível no espectro de sintomas que pode ir desde o ronco simples, a apnéia obstrutiva, até a síndrome da apnéia obstrutiva do sono. Em concordância com a literatura, encontrou-se prevalência de apnéia obstrutiva observada maior nos homens, com tendência de aumento com o avançar da idade e do IMC. ${ }^{5,14}$

Detectou-se associação entre ronco habitual e apnéia obstrutiva com tabagismo. Estudo populacional de Wetter et $\mathrm{al}^{20}$ realizado na Universidade de Wisconsin mostrou que tabagistas apresentavam maiores prevalências de ronco e distúrbios respiratórios do sono do que não tabagistas. Segundo esses autores, a redução da concentração de nicotina durante o sono dos fumantes poderia levar a uma instabilidade do sono, fator predisponente para obstrução da via aérea superior, ${ }^{20}$ com efeito dose-resposta, indicando que o risco aumenta com o consumo de cigarros. ${ }^{2,20} \mathrm{Em}$ um estudo multicêntrico, Franklin et $\mathrm{al}^{7}$ estimaram um risco atribuível ao tabagismo de $17,1 \%$ para ronco habitual e encontraram, além disso, uma associação de ronco habitual com tabagismo passivo.

Assim como Enright et al, ${ }^{5}$ o presente estudo encontrou associação de alcoolismo somente com ronco habitual, pois, após controle para o efeito do tabagismo, a associação com apnéia obstrutiva não foi confirmada. Entretanto, ao contrário dos homens, as mulheres alcoolistas tiveram risco 3,5 vezes maior de apresentaram apnéias obstrutivas do que as não alcoolistas. A ingestão aguda de álcool produz relaxamento nos músculos responsáveis pela permeabilidade da via aérea, alterando a respiração durante o sono. ${ }^{16}$ Sabe-se que o álcool aumenta marcadamente a freqüência e duração das apnéias nos indivíduos com síndrome da apnéia obstrutiva do sono. ${ }^{16}$ Em virtude da definição escolhida (dois ou mais pontos no escore $\mathrm{CAGE}^{6}$ ), a pesquisa identificou mulheres com uso abusivo de álcool expostas, provavelmente, mais constantemente aos seus efeitos agudos.

Os resultados do presente estudo permitem concluir que tanto a apnéia quanto o ronco têm prevalências elevadas na população estudada, semelhante às encontradas em outros estudos. Foram confirmadas associações com 
fatores não modificáveis, como sexo masculino e avanço da idade. Fatores de risco modificáveis como tabagismo, alcoolismo e obesidade mostraram associação significativa com ronco habitual. O progressivo envelhecimento da população e a crescente prevalência da obesidade apontam um possível incremento na prevalência dos sintomas relacionados ao sono. Portanto, deve ser estimulada a detecção precoce, por meio de programas de saúde com objetivo de identificar indivíduos com distúrbios respiratórios do sono.

\section{REFERÊNCIAS}

1. Sleep-related breathing disorders in adults: recommendations for syndrome definition and measurement techniques in clinical research. The Report of an American Academy of Sleep Medicine Task Force. Sleep. 1999;22(5):667-89.

2. Bloom JW, Kaltenborn WT, Quan SF. Risk factors in a general population for snoring. Importance of cigarette smoking and obesity. Chest. 1988;93(4):678-83.

3. Brooks LJ, Strohl KP. Size and mechanical properties of the pharynx in healthy men and women. Am Rev Respir Dis. 1992;146(6):1394-7.

4. Canani SF, John AB, Raymundi MG, Schönwald $S$, Menna Barreto SS. Prevalence of sleepiness in a group of Brazilian lorry drivers. Public Health. 2005;119(10):925-9.

5. Enright PL, Newman AB, Wahl PW, Manolio TA, Haponik EF, Boyle PJ. Prevalence and correlates of snoring and observed apneas in 5,201 older adults. Sleep. 1996;19(7):531-8.

6. Ewing JA. Detecting alcoholism. The CAGE questionnaire. JAMA. 1984;252(14):1905-7.

7. Franklin KA, Gíslason T, Omenaas E, Jõgi R, Jensen EJ, Lindberg $\mathrm{E}$, et al. The influence of active and passive smoking on habitual snoring. Am J Respir Crit Care Med. 2004;170(7):799-803.

8. Hoffstein V. Snoring. Chest. 1996;109(1):201-22.

9. Hoffstein V, Mateika S, Anderson D. Snoring: is it in the ear of the beholder? Sleep. 1994;17(6):522-6.

10. Katz I, Stradling J, Slutsky AS, Zamel N, Hoffstein V. Do patients with obstructive sleep apnea have thick necks? Am Rev Respir Dis. 1990;141(5 Pt 1):1228-31.

11. Koskenvuo M, Kaprio J, Telakivi T, Partinen M, Heikkilä K, Sarna S. Snoring as a risk factor for ischaemic heart disease and stroke in men. $\mathrm{Br}$ Med J (Clin Res Ed). 1987;294(6563):16-9.

12. Martinez D, Foppa M, Silva GC, Canais AA, Caramori C, Caleffi L. Sintomas respiratórios associados a distúrbios do sono em trabalhadores industriais do Rio Grande do Sul. J Pneumologia. 1994;20(3):127-32.

13. Norton PG, Dunn EV. Snoring as a risk factor for disease: an epidemiological survey. Br Med J (Clin Res Ed). 1985;291(6496):630-2.
14. Ohayon MM, Guilleminault C, Priest RG, Caulet M. Snoring and breathing pauses during sleep: telephone interview survey of a United Kingdom population sample. BMJ. 1997;314(7084):860-3.

15. Redline S, Kump K, Tishler PV, Browner I, Ferrette V. Gender differences in sleep disordered breathing in a community-based sample. Am / Respir Crit Care Med. 1994;149(3 Pt 1):722-6.

16. Robinson RW, Zwillich CW. The effect of drugs on breathing during sleep. Clin Chest Med. 1985;6(4):603-14.

17. Schmidt MDB, Tavares M, Polanczyk C, Pellanda L, Zimmer P. Validity of self-reported weight: a study of urban Brazilian adults. Rev Saude Publica. 1993;27(4):271-6.

18. Stores G, Crawford C. Medical student education in sleep and its disorders. J $R$ Coll Physicians Lond. 1998;32(2):149-53.

19. Torre-Bouscoulet L, Chávez E, Meza MS, Vázquez JC, Franco F, Muino A, et al. Snoring and Sleep-related Symptoms in Three Latin-American Cities. Proc Am Thorac Soc. 2005; 2:A767.

20. Wetter DW, Young TB, Bidwell TR, Badr MS, Palta M. Smoking as a risk factor for sleep-disordered breathing. Arch Intern Med. 1994;154(19):2219-24.

21. World Health Organization. Phisical status: the use and interpretation of anthropometry. Report of WHO Expert Committee. Geneva; 1995. (WHO Technical Reports Series, 854).

22. Young T, Finn L, Austin D, Peterson A. Menopausal status and sleep-disordered breathing in the Wisconsin Sleep Cohort Study. Am J Respir Crit Care Med. 2003;167(9):1181-5.

23. Young T, Palta M, Dempsey J, Skatrud J, Weber $\mathrm{S}$, Badr S. The occurrence of sleep-disordered breathing among middle-aged adults. N Engl J Med. 1993;328(17):1230-5.

24. Zielinski J, Zgierska A, Polakowska M, Finn L, Kurjata P, Kupsc W, et al. Snoring and excessive daytime somnolence among Polish middle-aged adults. Eur Respir J. 1999;14(4):946-50. 